\title{
Quadratic Cohomology
}

\author{
A. A. Agrachev
}

Received: 10 November 2014 / Accepted: 16 December 2014 / Published online: 24 December 2014 (C) Institute for Mathematical Sciences (IMS), Stony Brook University, NY 2014

\begin{abstract}
We study homological invariants of smooth families of real quadratic forms as a step towards a "Lagrange multipliers rule in the large" that intends to describe topology of smooth maps in terms of scalar Lagrange functions.
\end{abstract}

Keywords Lagrange multiplier · Quadratic form · Cohomology · Spectral sequence · Surgery

\section{Introduction}

Morse theory connects homology of Lebesgue sets and level sets of smooth real functions with critical points of the functions. The theory is based on a simple observation that a continuous deformation of the function does not influence the homotopy type of the level and Lebesgue sets for a prescribed value of the function as long as the value is not critical. Moreover, homology of the Lebesgue set is easier to control than one of the level set.

The same observation holds for level sets of smooth vector-functions. A natural generalization of a Lebesgue set is the space of solutions of a system of inequalities. The study of systems of inequalities and equations is partially reduced to the real functions case by the Lagrange multipliers rule. The Lagrange function of a vectorfunction $\left(\phi^{1}, \ldots, \phi^{k}\right)$ is a linear combination $p_{1} \phi^{1}+\cdots+p_{k} \phi^{k}, \sum_{i=1}^{k} p_{i}^{2}=1$, where the coefficients $p_{1}, \ldots, p_{k}$ of the linear combination are treated as extra variables, the

\footnotetext{
A. A. Agrachev (凶)

SISSA, Trieste, Italy

e-mail: agrachev@sissa.it

A. A. Agrachev

Steklov Mathematical Institute, Moscow, Russia
} 
Lagrange multipliers. The vector $0 \in \mathbb{R}^{k}$ is a critical value of $\left(\phi^{1}, \ldots, \phi^{k}\right)$ if and only if $0 \in \mathbb{R}$ is a critical value of the Lagrange function.

The title of the famous Marston Morse's book Morse (1934) is "The calculus of variations in the large". This paper is a step towards a Lagrange multipliers rule in the large. Our first observation, a starting point of the whole story, is that linearity with respect to the Lagrange multipliers is not important. More precisely, if two Lagrange functions

$$
f_{0}\left(p_{1}, \ldots, p_{k}, x\right)=\sum_{i=1}^{k} p_{i} \phi_{0}^{i}(x), \quad f_{1}\left(p_{1}, \ldots, p_{k}, x\right)=\sum_{i=1}^{k} p_{i} \phi_{1}^{i}(x)
$$

are connected by a homotopy $f_{t}, t \in[0,1]$, where $f_{t}$ are just smooth, not necessary linear with respect to the Lagrange multipliers and 0 is not a critical value of $f_{t}$ for all $t \in[0,1]$, then zero level sets of the vector functions $\left(\phi_{0}^{1}, \ldots, \phi_{0}^{k}\right)$ and $\left(\phi_{1}^{1}, \ldots, \phi_{1}^{k}\right)$ have equal homologies.

A similar property is valid for systems of inequalities; in this case Lagrange multipliers are taken from the intersection of the sphere with a convex cone. One inequality (like in Morse theory) corresponds to a point of the sphere. Actually, any point of the sphere of Lagrange multipliers represents a real function. We can think on usual homology of the space of solutions to the inequality as a kind of generalized cohomology of the point (different points may have different generalized cohomologies!). Similarly, the generalized cohomology of a convex subset of the sphere is the usual homology of the space of solutions to the corresponding system of inequalities. It is easy to extend the construction to more general subsets of the sphere like submanifolds with boundaries and corners. For the generalized cohomology to have good properties we impose some regularity conditions. In particular, not all convex subsets of the sphere are available but only those corresponding to regular systems of inequalities.

The generalized cohomology satisfies a natural modification of the EilenbergSteenrod axioms Eilenberg and Steenrod (1952). The most important "homotopy axiom" is based on the above property of the homologies of level sets when regular homotopies of the Lagrange functions are considered.

Such a cohomology theory is determined by the space of function $\operatorname{span}\left\{\phi^{1}, \ldots, \phi^{k}\right\}$; different spaces of functions give different generalized cohomologies. Moreover, as soon as a space of functions and the axioms are fixed we may try to find other cohomology theory that satisfies the same axioms but may be easier to compute. Such a theory should anyway have an intimate relation to the systems of inequalities and equations. The axioms imply that the cohomology of a point equals usual homology of space of solutions to the correspondent inequality; moreover, the cohomology of a convex set vanishes if the correspondent system of inequalities has no solutions.

This general setting is described in Sects. 2-4 of the paper. The main results are presented in Sects. 5, 6, where we build a cohomology theory that satisfies all the axioms in the case the space of functions is the space of quadratic forms. To compute the cohomology we define a spectral sequence $E^{r}$ (see Sect. 5) with clear explicit expressions for all the differentials. The homotopy invariance is proved in Sect. 6; the proof is based on the results of Agrachev (2011). 
The page $E^{2}$ and the differential $d_{2}$ of the spectral sequence $E^{r}$ are equal to the page $F^{2}$ and the differential $d_{2}$ of the spectral sequence $F^{r}$ described in Agrachev and Lerario (2012). The sequence $F^{r}$ converges to the homology of the space of solutions to the system of quadratic inequalities. We do not know higher differentials of the sequence $F^{r}$ and, for the moment, we do not see a reason for two spectral sequences to be equal. Anyway, this question remains open.

A couple of words on the differentials $d_{r}$ of the spectral sequence $E^{r}$. Recall that we deal with families of quadratic forms, i.e. symmetric matrices. Let $\lambda_{1}(p) \geq \cdots \geq$ $\lambda_{n}(p)$ be the eigenvalues of the matrix corresponding to the value $p$ of the parameter. A key role in the construction of the differentials is played by the cycles defined by the equations $\lambda_{i}(p)=\lambda_{i+1}(p)$ in the space of parameters. All differentials $d_{r}$ are some Massey operations involving these cycles, they are described in Sect. 5.

The following example shows a flavor of the developed theory and, in particular, the geometric meaning of the differential $d_{3}$. Let us consider the 3-dimensional space $i$ su(2) of Hermitian $2 \times 2$-matrices with zero trace. An Hermitian $2 \times 2$-matrix can be treated as a symmetric real $4 \times 4$-matrix commuting with the multiplication of the vectors in $\mathbb{C}^{2}=\mathbb{R}^{4}$ by the imaginary unit $i$. Thus $i \operatorname{su}(2) \subset \operatorname{Sym}\left(\mathbb{R}^{4}\right)$, where $\operatorname{Sym}\left(\mathbb{R}^{4}\right)$ is a 10 -dimensional space of real symmetric $4 \times 4$-matrices. Given a matrix $S \in \operatorname{Sym}\left(\mathbb{R}^{4}\right)$, let $\lambda_{1}(S) \geq \lambda_{2}(S) \geq \lambda_{3}(S) \geq \lambda_{4}(S)$ be its eigenvalues. If $S \in i \operatorname{su}(2)$, then $\lambda_{1}(S)=\lambda_{2}(S)=-\lambda_{3}(S)=-\lambda_{4}(S)$, i. e. the eigenvalues are double (the eigenspaces are complex lines). Recall that, in general, for an eigenvalue to be double is a codimension 2 property in $\operatorname{Sym}\left(\mathbb{R}^{4}\right)$.

Now take $S_{0} \in \operatorname{Sym}\left(\mathbb{R}^{4}\right)$ and translate the subspace $i \operatorname{su}(2)$ by $S_{0}$. We obtain an affine subspace $S_{0}+i \operatorname{su}(2) \subset \operatorname{Sym}\left(\mathbb{R}^{4}\right)$. Matrices from this affine subspace are not forced to be Hermitian and the eigenvalues are not necessary double. We set:

$$
C_{j}^{S_{0}}=\left\{H \in i \operatorname{su}(2): \lambda_{j}\left(S_{0}+H\right)=\lambda_{j+1}\left(S_{0}+H\right)\right\}, \quad j=1,2,3 .
$$

For generic $S_{0}, C_{j}^{S_{0}}$ are smooth real algebraic curves in the 3-dimensional space $i \operatorname{su}(2)$.

Proposition $C_{j}^{S_{0}}, j=1,2,3$, are not empty. Moreover, for generic $S_{0}$, the curve $C_{2}^{S_{0}}$ has odd linking numbers with $C_{1}^{S_{0}}$ and with $C_{3}^{S_{0}}$.

This proposition is proved in Sect. 7.

\section{Regular Homotopy}

Let $M$ be a smooth compact manifold. Given $\phi^{0}, \phi^{1} \ldots, \phi^{k} \in C^{1}(M)$, the system of equations $\phi^{0}(x)=\cdots=\phi^{k}(x)=0$ is regular if 0 is not a critical value of the map

$$
\varphi=\left(\phi^{0}, \ldots, \phi^{k}\right)^{T}: M \rightarrow \mathbb{R}^{k+1} .
$$

A homotopy $\varphi_{t}=\left(\phi_{t}^{0}, \ldots, \phi_{t}^{k}\right)^{T}$ is an isotopy of the system of equations $\phi_{t}^{0}=\cdots=$ $\phi_{t}^{k}=0$ if 0 is not a critical value of $\varphi_{t}, \forall t \in[0,1]$.

According to the standard Thom lemma, for any isotopy $\varphi_{t}$ there exists a family of diffeomorphisms $\Phi_{t}: M \rightarrow M, \Phi_{0}=i d$, such that 


$$
\varphi_{t}^{-1}(0)=\Phi_{t}\left(\varphi_{0}^{-1}(0)\right), \forall t \in[0,1]
$$

This is why one uses the term "isotopy". In particular, $\varphi_{1}^{-1}(0) \cong \varphi_{0}^{-1}, M \backslash \varphi_{1}^{-1} \cong$ $M \backslash \varphi_{0}^{-1}$.

Now consider the function $\varphi^{*}: S^{k} \times M \rightarrow \mathbb{R}$ defined by the formula $\varphi^{*}(p, x)=$ $\langle p, \varphi(x)\rangle$, where $p \in S^{k}=\left\{p \in \mathbb{R}^{k+1}:|p|=1\right\}$. It is easy to see that 0 is a critical value of $\varphi$ if and only if it is a critical value of $\varphi^{*}$.

Nothing prevents us from taking any function $f \in C^{1}\left(S^{k} \times M\right)$. We say that $f$ is regular if 0 is not a critical value of $f$. A homotopy $f_{t}, t \in[0,1]$, such that all $f_{t}$ are regular we call a regular homotopy. We have much more regular homotopies than isotopies. Nevertheless regular homotopy preserves an important information on the space of solutions to the system of equations.

Proposition 1 Assume that $f_{t}$ is a regular homotopy and $f_{0}=\varphi_{0}^{*}, f_{1}=\varphi_{1}^{*}$. Then $M \backslash \varphi_{0}^{-1}(0)$ is homotopy equivalent to $M \backslash \varphi_{1}^{-1}(0)$.

Proof We set

$$
B_{t}=\left\{(p, x) \in S^{k} \times M: f_{t}(p, x)>0\right\}
$$

Note that the projections $(p, x) \mapsto x$ restricted to $B_{0}$ and $B_{1}$ are fiber bundles over $M \backslash \varphi_{0}^{-1}(0)$ and $M \backslash \varphi_{1}^{-1}(0)$ whose fibers are hemispheres. In particular, $B_{0}$ is homotopy equivalent to $M \backslash \varphi_{0}^{-1}(0)$ and $B_{1}$ is homotopy equivalent to $M \backslash \varphi_{1}^{-1}(0)$.

We need the following Lemma.

Lemma 1 There exists a smooth family of diffeomorphisms $F_{t}: S^{k} \times M \rightarrow S^{k} \times M$ such that $F_{0}=i d, F_{t}\left(B_{0}\right) \subset B_{t}, \forall t \in[0,1]$.

Proof We set $z=(p, x) \in S^{k} \times M$ and look for a nonautonomous vector field $Z_{t}(z)$ such that the flow $F_{t}$ generated by the differential equation $\dot{z}=Z_{t}(z)$ has the desired property. It is sufficient to find a field $Z_{t}$ such that the equality $f_{t}(z)=0$ implies $\left\langle d_{z} f_{t}, Z_{t}(z)\right\rangle>0$. Moreover, it is sufficient to find such a field locally and then glue local pieces together by a partition of unity. It remains to mention that we can easily do it locally since 0 is not a critical value of $f_{t}$.

Lemma 1 implies that $B_{0}$ and $B_{1}$ are homotopy equivalent. Indeed, we can make a time substitution $t \mapsto 1-t$ and find a flow $G_{t}: S^{k} \times M \rightarrow S^{k} \times M$ such that $G_{t}\left(B_{1}\right) \subset B_{1-t}, G_{0}=i d$. The maps $G_{1} \circ F_{1}: B_{0} \rightarrow B_{0}$ and $F_{1} \circ G_{1}: B_{1} \rightarrow B_{1}$ are obviously homotopic to the identity.

Now I would like to extend the just described construction to systems of inequalities. As we'll see very soon, inequalities are very useful and helpful even if we are mainly interested in the equations. Let $K \subset \mathbb{R}^{k+1}$ be a closed convex cone. A system of inequalities is a relation $\varphi(x) \in K, x \in M$, were, as before, $\varphi=\left(\phi^{0}, \ldots, \phi^{k}\right)^{T}$. We say that the system of inequalities is regular (in the strong sense) if $\operatorname{im} D_{x} \varphi+K=$ $\mathbb{R}^{k+1}, \forall x \in \varphi^{-1}(K)$.

We take the dual cone $K^{\circ}=\left\{p \in \mathbb{R}^{k+1}:\langle p, y\rangle \leq 0, \forall y \in K\right\}$ and consider the "manifold with a convex boundary" $\left(K^{\circ} \cap S^{k}\right) \times M$. We say that a subset $V$ of a 
smooth manifold is a manifold with a convex boundary if $V$ is covered by coordinate neigborhoods whose intersections with $V$ are diffeomorphic to closed convex subsets of the Euclidean space. Smooth functions on the manifold with a convex boundary are restrictions of smooth functions on the ambient manifold. The tangent cone $T_{v} V$ is the closure of the set of velocities at $v$ of smooth curves starting from $v$ and contained in $V$.

Let $f: V \rightarrow \mathbb{R}$ be a $C^{1}$ function. We say that $v \in V$ is a critical point of $f$ if $\left\langle d_{v} f, \xi\right\rangle \leq 0, \forall \xi \in T_{v} V$.

Lemma 2 If the system of inequalities $\varphi(x) \in K$ is regular (in the strong sense), then 0 is not a critical point of $\left.\varphi^{*}\right|_{\left(K^{\circ} \cap S^{k}\right) \times M}$.

The proof is a straightforward check based on the duality $K^{\circ \circ}=K$; we leave it to the reader. The inverse statement is not true mainly due to the fact that $T_{y} K$ is, in general, bigger than $K$.

Definitions of regular functions on a manifold with a convex boundary and of regular homotopy for such functions are verbatim repetitions of the definitions for a manifold without boundary. An obvious modification of the proof of Proposition 1 gives:

Proposition 2 Assume that $f_{t}:\left(K^{\circ} \cap S^{k}\right) \times M \rightarrow \mathbb{R}, t \in[0,1]$, is a regular homotopy and $f_{0}=\left.\varphi_{0}^{*}\right|_{\left(K^{\circ} \cap S^{k}\right) \times M}, f_{1}=\left.\varphi_{1}^{*}\right|_{\left(K^{\circ} \cap S^{k}\right) \times M}$. Then $M \backslash \varphi_{0}^{-1}(K)$ is homotopy equivalent to $M \backslash \varphi_{1}^{-1}(K)$.

Remark Actually, the (obviously modified) proof of Proposition 1 gives more; namely, under conditions of Proposition 2 the inclusion

$$
\left(t, B_{t}\right) \hookrightarrow \bigcup_{\tau \in[0,1]}\left(\tau, B_{\tau}\right)
$$

of the subspaces of $[0,1] \times V \times M$ is a homotopy equivalence, $\forall t \in[0,1]$.

So the homotopy type of the complement to the space of solutions to the system of inequalities is preserved by regular homotopies. It happens that homology of the space of solutions is preserved as well.

Proposition 3 Assume that $f_{t}:\left(K^{\circ} \cap S^{k}\right) \times M \rightarrow \mathbb{R}, t \in[0,1]$, is a regular homotopy and $f_{0}=\left.\varphi_{0}^{*}\right|_{\left(K^{\circ} \cap S^{k}\right) \times M}, f_{1}=\left.\varphi_{1}^{*}\right|_{\left(K^{\circ} \cap S^{k}\right) \times M}$. Then the homology groups of $\varphi_{0}^{-1}(K)$ and $\varphi_{1}^{-1}(K)$ with coefficients in a field are isomorphic.

Proof We start from the case $K \neq-K$, i.e. $K$ is not a subspace and the system of inequalities is not just a system of equations. In this case, $K^{\circ} \cap S^{k}$ is contractible and we have the following series of homotopy equivalences of the pairs:

$$
\begin{aligned}
& \left(M, M \backslash \varphi_{0}^{-1}(K)\right) \sim\left(\left(K^{\circ} \cap S^{k}\right) \times M, B_{0}\right) \sim\left(\left(K^{\circ} \cap S^{k}\right) \times M, B_{1}\right) \\
& \quad \sim\left(M, M \backslash \varphi_{1}^{-1}(K)\right)
\end{aligned}
$$


where $B_{t}=\left\{(p, x) \in\left(K^{\circ} \cap S^{k}\right) \times M: f_{t}(p, x)>0\right\}$ (see the proof of Proposition 1). Hence $H^{*}\left(M, M \backslash \varphi_{0}^{-1}(K)\right) \cong H^{*}\left(M, M \backslash \varphi_{1}^{-1}(K)\right)$. Alexander-Pontryagin duality completes the proof for this case.

The case of a system of equations is easily reduced to the case just studied if we add the tautological inequality $1 \geq 0$ to the system. Let us explain this in more detail. If $K$ is a subspace, then we may assume without lack of generality that $K=0$. Now extend the function $f_{t}$ to $\mathbb{R}^{k+1} \times M$ as a degree one homogeneous function with respect to the variable $p$ (keeping the symbol $f_{t}$ for the extension) and consider the functions

$$
\bar{f}_{t}:(p, v, x) \mapsto f_{t}(p, x)+v, \quad|p|^{2}+v^{2}=1, v \leq 0 .
$$

It is easy to see that $\bar{f}_{t}$ are regular. To be absolutely rigorous, we have to smooth out $f_{t}$ at the points $(0, x)$ but, in fact, nothing depends on the way we do it because $\bar{f}_{t}$ is far from 0 at these points.

\section{Localization}

Let $V$ be a manifold with a convex boundary and $f: V \times M \rightarrow \mathbb{R}$ a $C^{1}$-function. In this section, we assume that $M$ is a real-analytic manifold and $f(v, \cdot)$ is a subanalytic function, $\forall v \in V$. It is convenient to think about $f$ as a family of subanalytic functions $f(v, \cdot)$ on $M$ which depends on the parameter $v \in V$, and we introduce the notation $f_{v} \doteq f(v, \cdot)$. "Localization" in this section is the localization with respect to the parameter $v$; the variable $x \in M$ remains global.

We say that the family $f_{v}$ is regular at $v_{0} \in V$ if the set $\left\{v_{0}\right\} \times f_{v_{0}}^{-1}(0)$ does not contain critical points of $f$.

Proposition 4 Assume that the family $f_{v}, v \in V$, is regular at $v_{0} \in V$. Then $v_{0}$ has a compact neighborhood $O_{v_{0}}$ and centered at $v_{0}$ local coordinates $\Phi$ such that $U_{0} \doteq \Phi\left(O_{v_{0}}\right)$ is convex and the function $\left.(f \circ \Phi+t)\right|_{\varepsilon U_{0} \times M}$ is regular for any sufficiently small nonnegative constants $t, \varepsilon$ one of which is strictly positive.

Proof We may assume that $v_{0}=0$ is the origin of a Euclidean space and $\Phi=i d$. Given $a \in C^{1}(M), y \in \mathbb{R}$, we set $C_{a}(y)=\left\{x \in a^{-1}(y): d_{x} a=0\right\}$ If $0 \in \mathbb{R}$ is not a critical value of $f_{0}$, i.e. $C_{f_{0}}=\emptyset$, then the statement is obvious; otherwise, for any $x \in C_{f_{0}}$ there exists $v_{x} \in U_{0}$ such that $\left\langle\frac{\partial f}{\partial v}(0, x), v_{x}\right\rangle \geq \alpha>0$, where $\alpha$ is a positive constant. Then, by the continuity, there exists $\delta>0$ such that for any $\tau \in[-\delta, \delta], v \in \delta U_{0}, x \in C_{f_{v}}(\tau)$, there exists $\hat{x} \in C_{f_{0}}(0)$ such that

$$
\left\langle\frac{\partial f}{\partial v}(v, x), v_{\hat{x}}\right\rangle \geq \delta>0
$$

Now let $v \in \varepsilon U_{0}, t \in[0, \delta]$, and $x \in C_{f_{v}}(-t)$; then $d_{x} f_{v}=0$ and $\left|d_{x} f_{0}\right| \leq c \varepsilon$ for some constant $c$. We have:

$$
f(0, x)=f(v, x)-\left\langle\frac{\partial f}{\partial v}(v, x), v\right\rangle+o(\varepsilon),
$$


where $\frac{o(\varepsilon)}{\varepsilon} \rightarrow 0$ as $\varepsilon \rightarrow 0$ uniformly for all $v \in \varepsilon U_{0}, x \in C_{f_{0}}(-t), t \in[0, \delta]$. Then

$$
\left\langle\frac{\partial f}{\partial v}(v, x),-v\right\rangle=t+f_{0}(x)-o(\varepsilon)
$$

and, according to (1),

$$
\left\langle\frac{\partial f}{\partial v}(v, x), \varepsilon v_{\hat{x}}-v\right\rangle \geq t+f_{0}(x)+\varepsilon \delta-o(\varepsilon) .
$$

The Lojasevic inequality Kurdyka (1998) gives:

$$
\left|f_{0}(x)\right| \leq c^{\prime}\left|d_{x} f_{0}\right|^{1+\rho} \leq c^{\prime} c^{1+\rho} \varepsilon^{1+\rho},
$$

where $c^{\prime}, \rho$ are positive constants. Hence $\left\langle\frac{\partial f}{\partial v}(v, x), \varepsilon v_{\hat{x}}-v\right\rangle>0$ if $\varepsilon$ is sufficiently small.

Corollary 1 Let $V$ be a compact convex set, $0 \in V$. Assume that the family $f_{v}, v \in V$, is regular at 0 . Then for any sufficiently small $\varepsilon>0$ the homotopy

$$
(t, v, x) \mapsto f(t v, x)+(1-t) \varepsilon, \quad t \in[0,1], v \in \varepsilon V, x \in M
$$

between $\left.f\right|_{\varepsilon V \times M}$ and the constant family $(v, x) \mapsto f(0, x)+\varepsilon$ is regular.

\section{A Cohomology Theory}

Let $M$ be a real-analytic manifold and $\mathcal{A} \subset C^{1}(M)$ a set of subanalytic functions. Let $W \subset V$ be a pair of manifolds with convex boundaries and $f: V \times M \rightarrow \mathbb{R}$ a regular function such that $f_{v} \in \mathcal{A}, \forall v \in V$, and $\left.f\right|_{W \times M}$ is also regular.

We set $B_{f}=\{(v, x): v \in V, f(v, x)>0\}$ and define

$$
H_{\mathcal{A}}\left(f_{V}, f_{W}\right) \doteq H^{\cdot}\left(V \times M,(W \times M) \cup B_{f}\right), \quad H_{\mathcal{A}}(f) \doteq H_{\mathcal{A}}\left(f_{V} \cdot f_{\emptyset}\right)
$$

The pairs of regular functions $\left(f_{V}, f_{W}\right)$ form a category $\mathfrak{F}_{\mathcal{A}}$ with morphisms $\varphi^{*}$ : $\left(f_{V_{0}}^{0}, f_{W_{0}}^{0}\right) \mapsto\left(f_{V_{1}}^{1}, f_{W_{1}}^{1}\right)$, where $\varphi: V_{1} \rightarrow V_{0}$ is a $C^{1}$-map such that $\varphi\left(W_{1}\right) \subset W_{0}$ and $f_{v}^{1}=f_{\varphi(v)}^{0}, \forall v \in V_{1}$. Then $H_{\mathcal{A}}$ is a functor from this category to the category of commutative groups.

This is a kind of cohomology functor which satisfies natural modifications of the Steenrod-Eilenberg axioms except for the dimension axiom. The exactness and excision are obvious and we do not repeat them. Homotopy axiom deals with $f:[0,1] \times V \times M \rightarrow \mathbb{R}$ such that $f_{\{t\} \times V} \in \mathfrak{F}_{\mathcal{A}}, \forall t \in[0,1]$, and claims that the inclusions $\{t\} \times V \hookrightarrow[0,1] \times V, t \in[0,1]$, induce the isomorphisms of cohomology groups:

$$
\left.H_{\mathcal{A}}\left(f_{[0,1] \times V}, f_{[0,1] \times W}\right) \cong H_{\mathcal{A}}\left(f_{\{t\} \times V}, f_{\{t\} \times W}\right)\right)
$$


This simple but not totally obvious fact was explained in Sect. 2.

The dimension axiom is substituted by the following one: if $V=\{v\}$ is a point then

$$
H_{\mathcal{A}}\left(f_{\{v\}}\right)=H^{\cdot}\left(M,\left\{x \in M: f_{v}(x)>0\right\}\right) .
$$

The "points" for us are regular elements of $\mathcal{A}$ and different points may have different cohomology.

Standard singular cohomology is a special case. Indeed, let the set $\mathcal{A}$ consist of one point, $\mathcal{A}=\{a\}$, and $a(x)<0, \forall x \in M$. We have:

$$
H_{\{a\}}(V, W)=H^{\cdot}(V, W) \times H^{\cdot}(M) .
$$

Now assume that $\mathcal{A}+t \subset \mathcal{A}$ for any nonnegative constant $t$. Given a map $v \mapsto f_{v}$ from $V$ to $\mathcal{A}$ we denote by $(f+t)_{[0, c] \times V}$ the map $(t, v) \mapsto f_{v}+t, t \in[0, c], v \in V$. It was proved in Sect. 3 that for any $v \in V$ there exists a neigborhood $U_{v} \subset V$ and $\varepsilon>0$ such that the inclusions

$$
U_{v} \times\{0\} \hookrightarrow U_{v} \times[0, \varepsilon], \quad\{v\} \times\{\varepsilon\} \hookrightarrow U_{v} \times[0, \varepsilon]
$$

induce the isomorphisms of the cohomology groups

$$
H_{\mathcal{A}}\left(f_{U_{v}}\right) \cong H_{\mathcal{A}}\left((f+t)_{[0, \varepsilon] \times U_{v}}\right) \cong H_{\mathcal{A}}\left(f_{\{v\}}+\varepsilon\right)
$$

In other words, cohomology of a "small neighborhood" is equal to the cohomology of a "point".

Now assume that the cohomology are taken with coefficients in a field and that $\operatorname{dim} M=n$. Then the cohomology of a "point"

$$
H_{\mathcal{A}}^{i}\left(f_{\{v\}}\right)=H^{i}\left(M,\left\{x \in M: f_{v}(x)>0\right\}\right)=H_{n-i}\left(\left\{x \in M: f_{v}(x) \leq 0\right\}\right),
$$

$0 \leq i \leq n$, is simply usual homology of the space of solutions to the inequality $f_{v}(x) \leq 0$.

The localization at a point plus the algebraic homology machinery (based on the axioms) gives a good chance to recover the usual homology of the space of solutions of a system of inequalities from the ones of the individual inequalities of the form $a(x) \leq 0$, where $a \in \mathcal{A}$. The success is somehow guaranteed if $H_{\mathcal{A}}$ is a unique cohomology theory for $\mathcal{A}$ that satisfies the described axioms. On the other hand, any other cohomology theory that satisfies the same axioms gives additional important invariants of systems of inequalities or equations for functions from $\mathcal{A}$.

Let me explain it better for regular systems of equations

$$
\phi^{0}(x)=\cdots=\phi^{k}(x)=0, \quad \phi^{i} \in \mathcal{A}, i=0,1, \ldots, k
$$


An isotopy $\varphi_{t}=\left(\phi_{t}^{0}, \ldots, \phi_{t}^{k}\right)^{T}, t \in[0,1]$, of such systems is called $\mathcal{A}$-rigid if $\phi_{t}^{i} \in \mathcal{A}$ for all $t \in[0,1]$. In this case, $\varphi_{t}^{*} \in \mathfrak{F}_{\mathcal{A}}$, where, recall,

$$
\varphi_{t}^{*}: S^{k} \times M \rightarrow \mathbb{R}, \quad \varphi_{t}^{*}(p, x)=\left\langle p, \varphi_{t}(x)\right\rangle .
$$

Let $\hat{H}_{\mathcal{A}}$ be a cohomology functor that satisfies our axioms; then, according to the homotopy axiom, $\hat{H}_{\mathcal{A}}\left(\varphi_{0}^{*}\right)=\hat{H}_{\mathcal{A}}\left(\varphi_{1}^{*}\right)$. In other words, $\hat{H}_{\mathcal{A}}$ is an invariant of the $\mathcal{A}$ rigid isotopy. Moreover, it is an invariant of regular homotopies in $\mathfrak{F}_{\mathcal{A}}$ that are much more general than $\mathcal{A}$-rigid isotopies.

Let $\varphi=\left(\phi^{0}, \ldots, \phi^{k}\right)^{T},(\nu, p) \in \mathbb{R} \times \mathbb{R}^{k+1}, x \in M$; we set $\bar{\varphi}^{*}(\nu, p, x)=$ $v+\langle p, \varphi(x)\rangle$ and denote by $S_{-}^{k+1}$ the low semi-sphere in $\mathbb{R} \times \mathbb{R}^{k+1}, S_{-}^{k+1}=\{(v, p)$ : $\left.v \leq 0, v^{2}+|p|^{2}=1\right\}$.

Proposition 5 If $\varphi^{-1}(0)=\emptyset$, then $\hat{H}_{\mathcal{A}}\left(\bar{\varphi}_{S_{-}^{k+1}}^{*}\right)=0$.

Proof Let $c \in \mathbb{R}, B_{c}^{k+1}=\left\{(c, p): p \in \mathbb{R}^{k+1},|p| \leq 1\right\}$. Note that $\left.\bar{\varphi}^{*}\right|_{B_{c}^{k+1} \times M}$ is a regular function for any $c>0$ (this is true for any smooth map $\varphi: M \rightarrow \mathbb{R}^{k+1}$ ). Moreover, $\bar{\varphi}_{B_{c}^{k+1}}^{*}$ is regularly homotopic in $\mathfrak{F}_{\mathcal{A}}$ to the constant function $c$; indeed, the homothety of the ball $B_{c}^{k+1}$ to its center along the radii provides us with the desired regular homotopy. Hence $\hat{H}_{\mathcal{A}}\left(\bar{\varphi}_{B_{c}^{k+1}}^{*}\right)=0$.

The function $\left.\bar{\varphi}^{*}\right|_{B_{0}^{k+1} \times M}$ is regular if and only if $\varphi^{-1}(0)=\emptyset$. If it is regular, then it is regularly homotopic to $\left.\bar{\varphi}^{*}\right|_{B_{c}^{k+1} \times M}$, where $c>0$, and $H_{\mathcal{A}}\left(\bar{\varphi}_{B_{0}^{k+1}}^{*}\right)=0$. It remains to note that the homotopy between $\bar{\varphi}_{B_{0}^{k+1}}^{*}$ and $\bar{\varphi}_{S_{-}^{k+1}}^{*}$ induced by the homotopy $(t ; v, p) \mapsto((1-t) v, p), t \in[0,1],(v, p) \in S_{-}^{k+1}$, is also regular.

Let $M=\mathbb{R} P^{N}=\left\{(x,-x): x \in S^{k}\right\}$ and $\mathcal{Q}(N)$ the space of real quadratic forms on $\mathbb{R}^{N+1}$ treated as functions on $\mathbb{R} P^{N}$. The main goal of this paper is to construct a cohomology theory $\hat{H}_{\mathcal{Q}(N)}$. This is not just an abstract construction: we give an effective way to compute the cohomology.

In what follows all cochains and cohomologies are with coefficients in $\mathbb{Z}_{2}$. We omit the symbol $\mathbb{Z}_{2}$ to simplify notations.

\section{A Spectral Sequence}

Now we focus on the space $\mathcal{Q}(N)$ with fixed $N$ and omit the $\operatorname{argument} N$ in order to simplify notations. We denote by the same symbol a quadratic form on $\mathbb{R}^{N+1}$ and the function on $\mathbb{R} P^{N}$ induced by this form. A quadratic form $q$ induces a regular function on $\mathbb{R} P^{N}$ if and only if $\operatorname{ker} q=0$. More precisely, critical points of $q: \mathbb{R} P^{N} \rightarrow \mathbb{R}$ at $q^{-1}(0)$ are exactly $\bar{x}=(x,-x) \in \mathbb{R} P^{N}$ such that $x \in \operatorname{ker} q \cap S^{N}$.

Some notations. Let $\lambda_{1}(q) \geq \cdots \geq \lambda_{N+1}(q)$ be the eigenvalues of the symmetric operator associated to the quadratic form $q \in \mathcal{Q}$. We set 


$$
\begin{gathered}
\Lambda_{j, m}=\left\{q \in \mathcal{Q}: \lambda_{j-1}(q) \neq \lambda_{j}(q)=\lambda_{j+m-1}(q) \neq \lambda_{j+m}(q)\right\}, \\
\Lambda_{j, m}^{0}=\left\{q \in \Lambda_{j, m}: \lambda_{j}(q)=0\right\},
\end{gathered}
$$

$j=1, \ldots, N, m=2, \ldots, N-j+2$. It is well-known that $\Lambda_{j, m}$ is a smooth submanifold of codimension $\frac{m(m+1)}{2}-1$ in $\mathcal{Q}$ while $\Lambda_{j, m}^{0}$ is a codimension 1 submanifold of $\Lambda_{j, m}$ (see Agrachev 2011, Prop. 1 for a short proof).

We say that the pair $\left(f_{V}, f_{W}\right) \in \mathfrak{F}_{\mathcal{Q}}$ is in general position if the boundaries $\partial V, \partial W$ are smooth and the map $v \mapsto f_{v}, v \in V$, as well as the restrictions of this map to $W, \partial V, \partial W$ are transversal to $\Lambda_{j, m}$ and $\Lambda_{j, m}^{0}$, for $j=1, \ldots, N, m=2, \ldots, N-$ $j+2$.

It is sufficient to construct $\hat{H}\left(f_{V}, f_{W}\right)$ and check the axioms for the pairs in general position. Indeed, if the the boundaries $\partial V, \partial W$ are smooth, then standard transversality arguments allow to approximate any pair by a pair in the general position. Moreover, any two sufficiently close approximations are regularly homotopic and have equal cohomology $\hat{H}$ according to the homotopy axiom. The cohomology of the given pair is equal, by definition, to the cohomology of a sufficiently close approximation in general position.

Similar arguments work in the case of nonsmooth boundaries. Given a manifold $V$ with a convex boundary we can always find a smooth vector field transversal to the boundary $\partial V$. Trajectories of this field passing through $\partial V$ provide us with a tubular neighborhood of the boundary. Smooth sections of the tubular neighborhood give us smooth approximations of $\partial V$ inside $V$ and we obtain $\tilde{V} \subset V$, where $\partial \tilde{V}$ is a smooth approximation of $\partial V$. The approximation is good if the time to move from $\partial \tilde{V}$ to $\partial V$ along trajectories of our transversal vector field is a $C^{0}$-small semi-concave function with a uniformly bounded differential (recall that the differential is defined almost everywhere).

It is easy to see that $\left(f_{\tilde{V}}, f_{\tilde{W}}\right) \in \mathfrak{F}_{\mathcal{Q}}$ for any sufficiently good approximation $\tilde{W} \subset W, \tilde{W} \subset \tilde{V} \subset V$. Moreover, natural diffeomorphisms of different tubular neighborhoods induce diffeomorphisms homotopic to the identity of good approximations $(\tilde{V}, \tilde{W})$ and natural isomorphisms of cohomologies $\hat{H}\left(f_{\tilde{V}}, f_{\tilde{W}}\right)$. The cohomology $\hat{H}\left(f_{V}, f_{W}\right)$ is equal, by definition, to $\hat{H}\left(f_{\tilde{V}}, f_{\tilde{W}}\right)$, where $(\tilde{V}, \tilde{W})$ is a sufficiently good approximation of $(V, W)$ by the pair of manifolds with smooth boundaries.

Let $f: V \rightarrow \mathcal{Q}, f \in \mathfrak{F}_{\mathcal{Q}}$, be in general position ${ }^{1}$ and

$$
V_{f}^{j}=\left\{v \in V: \lambda_{j}(f(v))>0\right\}, \quad j=1, \ldots, N+1,
$$

a decreasing filtration of $V$ by open subsets. We equip $V$ with a Riemannian metric and take $\varepsilon>0$ so small that $V_{f}^{j}$ and $f^{-1}\left(\Lambda_{j, m}\right)$ are homotopy retracts of their radius $(\operatorname{dim} V) \varepsilon$ neighborhood, $j=1, \ldots, N+1, m=2, \ldots, N-j+2$.

Now consider a smooth singular simplex $\varsigma: \Delta^{i} \rightarrow V$, where $\Delta^{i}$ is the standard $i$-dimensional simplex. We say that $\varsigma$ is adapted to $f$ if the diameter of $\varsigma\left(\Delta^{i}\right)$ is

\footnotetext{
${ }^{1}$ For simplicity, we keep the symbol $f$ for the map $v \mapsto f_{v}$.
} 
smaller than $\varepsilon$ and the restriction of $f \circ \varsigma$ to any face $D$ of $\Delta^{i}$ satisfies the following properties:

(i) $\left.f \circ \varsigma\right|_{D} \pitchfork \Lambda_{j, m}$;

(ii) if $\operatorname{dim} D=4$ and $f \circ \varsigma(D) \cap \Lambda_{j, 2} \neq \emptyset$ then $f \circ \varsigma(D) \cap \Lambda_{j+1,2}=f \circ \varsigma(D) \cap$ $\Lambda_{j-1,2}=\emptyset, j=1, \ldots, N$.

Manifold $V$ admits a triangulation by adapted simplices. The more delicate property (ii) can be achieved because $\bar{\Lambda}_{j, 2} \cap \bar{\Lambda}_{j+1,2}=\bar{\Lambda}_{j, 3}$ has codimension 5 in $\mathcal{Q}$.

We denote by $C_{f, i}(V)$ the space of $i$-dimensional singular chains in $V$ generated by the adapted singular simplices with coefficients in $\mathbb{Z}_{2}$. Let $U$ be an open subset of $V$; then $C_{f, i}(U)$ is a subspace of $C_{f, i}(V)$ generated by singular simplices with values in $U$ and $C_{f}^{i}(V, U)$ is the space of linear forms on $C_{f, i}(V)$ that vanish on $C_{f, i}(U)$. We obtain a cochain complex

$$
\ldots \rightarrow C_{f}^{i-1}(V, U) \stackrel{\delta}{\longrightarrow} C_{f}^{i}(V, U) \stackrel{\delta}{\longrightarrow} C_{f}^{i+1}(V, U) \rightarrow \ldots,
$$

where $\delta$ is usual coboundary of singular cochains. The cohomology of the complex (2) coincides with standard cohomology of the pair $(V, U)$ with coefficients in $\mathbb{Z}_{2}$ : $\operatorname{ker} \delta / \operatorname{im} \delta=H^{\cdot}(V, U)$.

We define cocycles $l_{f}^{j} \in C_{f}^{2}(V), \quad j=1, \ldots, N$ as follows: given a singular simplex $\varsigma \in C_{f, 2}(V), l_{f}^{j}(\varsigma)$ is the intersection number modulo 2 of $f \circ \varsigma$ and $\Lambda_{j, 2}$. We have: $l_{f}^{j} \smile l_{f}^{j+1}=0, \quad j=1, \ldots, N-1$. Here $\smile$ is the cup product of singular cochains. The maps $\ell_{j}: \varsigma \rightarrow \varsigma \smile l_{f}^{j}$ define homomorphisms $\ell_{j}: C_{f}^{i}(V, U) \rightarrow$ $C_{f}^{i+2}(V, U)$. We have $\delta \circ \ell_{j}=\ell_{j} \circ \delta, \ell_{j} \circ \ell_{j+1}=0$.

Given $\tau>0$ let $V_{f}^{j}(\tau)$ be the radius $\tau$ neighborhood of $V_{f}^{j}$. We set:

$$
C_{j}^{i}(f)=C_{f}^{i}\left(V, V_{f}^{j}(i \varepsilon)\right), \quad C^{n}(f)=\bigoplus_{i+j=n} C_{j+1}^{i}(f)
$$

then $\ell_{j}\left(C_{j+1}^{i}(f)\right) \subset C_{j}^{i+2}(f)$. Finally, we define the differential $d: C^{n}(f) \rightarrow$ $C^{n+1}(f)$ by the formula $\left.d\right|_{C_{j-1}^{i}}(f)=\delta+\ell_{j}$.

The cohomology $\hat{H}_{\mathcal{Q}}(f)$ is, by definition, the cohomology of the complex

$$
\ldots \rightarrow C^{n-1}(f) \stackrel{d}{\longrightarrow} C^{n}(f) \stackrel{d}{\longrightarrow} C^{n+1}(f) \rightarrow \ldots
$$

Remark A pedantic reader would say that the cochain groups $C^{n}(f)$ depend on the small parameter $\varepsilon$. It is not hard to see that the cohomologies of complex (3) for different $\varepsilon$ are naturally isomorphic.

Consider a filtration of the complex $\bigoplus_{n \geq 0} C^{n}(f)=\bigoplus_{n \geq 0} \bigoplus_{i \geq 0} C_{n-i+1}^{i}(f)$ by subcomplexes $\bigoplus_{n \geq 0} \underset{i \geq \alpha}{\bigoplus} C_{n-i+1}^{i}(f), \alpha=0,1, \ldots, \operatorname{dim} V$ and the spectral sequence $E_{i, j}^{r}$ of this filtration converging to $\hat{H}_{\mathcal{Q}}(f)$. We have: 


$$
E_{i, j}^{1}=C_{j+1}^{i}(f), \quad d_{1}: C_{j+1}^{i}(f) \rightarrow C_{j+1}^{i+1}(f), d_{1}=\delta .
$$

Hence

$$
E_{i j}^{2}=H^{i}\left(V, V_{f}^{j+1}\right), \quad d_{2}: H^{i}\left(V, V_{f}^{j+1}\right) \rightarrow H^{i+2}\left(V, V_{f}^{j}\right)
$$

Moreover, the differential (4) is induced by $\ell_{j}$ and has a very simple explicit expression. Namely, let $\bar{l}_{f}^{j} \in H^{2}\left(V, V \backslash f^{-1}\left(\bar{\Lambda}_{j, 2}\right)\right)$ be the cohomology class of the cocycle $l_{f}^{j}$. Then $d_{2}$ is the composition of the map

$$
\bar{\ell}_{j}: H^{i}\left(V, V_{f}^{j+1}\right) \rightarrow H^{i+2}\left(V, V_{f}^{j+1} \cup\left(V \backslash f^{-1}\left(\bar{\Lambda}_{j, 2}\right)\right)\right)
$$

defined by the formula $\bar{\ell}_{j}(x)=x \smile \bar{l}_{f}^{j}, x \in H^{i}\left(V, V_{f}^{j+1}\right)$, and the homomorphism $H^{i+2}\left(V, V_{f}^{j+1} \cup\left(V \backslash f^{-1}\left(\bar{\Lambda}_{j, 2}\right)\right)\right) \rightarrow H^{i+2}\left(V, V_{f}^{j}\right)$ induced by the inclu$\operatorname{sion} V_{f}^{j} \subset V_{f}^{j+1} \cup\left(V \backslash f^{-1}\left(\bar{\Lambda}_{j, 2}\right)\right)$.

We see that $E_{i, j}^{2}$ and $d_{2}$ coincide with the second page $F_{i, j}^{2}$ and the differential $d_{2}: F_{i, j}^{2} \rightarrow F_{i+2, j-1}^{2}$ of the spectral sequence converging to $H_{\mathcal{Q}}(f)$ studied in Agrachev and Lerario (2012) (see Theorems 25 and 28 of the cited paper). Hence $E_{i, j}^{3}=F_{i, j}^{3}$.

Now we are going to give simple explicit expressions for all differentials $d_{r}$ : $E_{i, j}^{r} \rightarrow E_{i+r, j-r+1}^{r}, r \geq 3$.

Let $\xi \in C_{j+1}^{i}(f)=E_{i, j}^{1}$ be a $\delta$-cocycle such that its cohomology class $\bar{\xi} \in$ $H^{i}\left(V, V_{f}^{j+1}\right)=E_{i, j}^{2}$ is a $d_{2}$-cocycle. Then $\xi \smile l_{f}^{j}=\delta \eta$, where $\eta \in C_{j}^{i+1}(f)$. Moreover, $d_{3}(\bar{\xi})$ is the cohomology class of $\eta \smile l_{f}^{j-1}$ in $H^{i+3}\left(V, V_{f}^{j-1}\right)$ modulo $d_{2-}$ coboundaries while $l_{f}^{j} \smile l_{f}^{j-1}=0$. Hence $d_{3}(\bar{\xi})$ is the Massey product $\left\langle\bar{\xi}, \bar{l}_{f}^{j}, \bar{l}_{f}^{j-1}\right\rangle$ combined with an appropriate inclusion homomorphism (see McCleary 2001, Ch. 8 for the definition and basic properties of Massey products).

Now assume that $\xi$ survives in $E_{i, j}^{r}$, i. e. classes of $\xi$ are cocycles for $d_{3}, \ldots, d_{r-1}$. The induction procedure implies that $d_{r}(\xi)$ is the $r$-fold Massey product $\left\langle\bar{\xi}, \bar{l}_{f}^{j}, \ldots, \bar{l}_{f}^{j-r+2}\right\rangle$ combined with appropriate inclusion homomorphisms.

Indeed, since the class of $\xi$ is $d_{r-1}$-cocycle then, according to the induction assumption, $\left\langle\bar{\xi}, \bar{l}_{f}^{j}, \ldots, \bar{l}_{f}^{j-r+3}\right\rangle \ni \delta \zeta$, where $\zeta \in C_{j-r+3}^{i+r-2}(f)$, and $d_{r}(\bar{\xi})$ is the class of $\zeta \smile l_{f}^{j-r+2}$.

If $\operatorname{dim} V \leq k$, then $E_{i, j}^{2}=0$ for $i>k$. In particular, if $\operatorname{dim} V=3$ then the last possibly nontrivial differential is $d_{3}$. This differential has a clear geometric meaning that we are going to describe. Assume that $H_{1}\left(V ; \mathbb{Z}_{2}\right)=0$ and $\partial V$ is connected or empty (the three-dimensional sphere and ball are available). Then $H_{2}\left(V ; \mathbb{Z}_{2}\right)=0$ and the linking number mod 2 of a 1-dimensional cycle in $V$ with a 1-dimensional cycle in $(V, \partial V)$ are well-defined. We have:

$$
d_{3}: H^{0}\left(V, V_{f}^{j+1}\right) \longrightarrow H^{3}\left(V, V_{f}^{j-1}\right) .
$$


Moreover, ranks of $H^{0}\left(V, V_{f}^{j+1}\right)$ and $H^{3}\left(V, V_{f}^{j-1}\right)$ are either one or zero.

If both ranks are equal to one, then $d_{3}$ sends the generator of $H^{0}\left(V, V_{f}^{j+1}\right)$ to the generator of $H^{3}\left(V, V_{f}^{j-1}\right)$ multiplied by the linking number of 1-dimensional cycles $f^{-1}\left(\Lambda_{j, 2}\right)$ and $f^{-1}\left(\Lambda_{j-1,2}\right)$, according to the direct implementation of the above construction.

Let $W \subset V$ be such that the pair $\left(f_{V}, f_{W}\right) \in \mathfrak{F}_{\mathcal{Q}}$ is in general position and $\tilde{W} \supset W$ be an appropriate tubular neighborhood of $W$ such that the pairs $\left(W, W_{f}^{j}\right)$ are homotopy retracts of $\left(\tilde{W}, \tilde{W}_{f}^{j}\right)$ and $\hat{H}^{\cdot}\left(f_{\tilde{W}}\right)$ is naturally isomorphic to $\hat{H}^{\cdot}\left(f_{\tilde{W}}\right)$. We define:

$$
C_{j}^{i}\left(f_{V}, f_{W}\right) \doteq C_{j}^{i}\left(f_{V}\right) \cap C_{f}^{i}(V, \tilde{W}), \quad C^{n}\left(f_{V}, f_{W}\right)=\bigoplus_{i+j=n} C_{j+1}^{i}\left(f_{V}, f_{W}\right)
$$

The cohomology $\hat{H}_{\mathcal{Q}}\left(f_{V}, f_{W}\right)$ is, by definition, the cohomology of the complex

$$
\ldots \rightarrow C^{n-1}\left(f_{V}, f_{W}\right) \stackrel{d}{\longrightarrow} C^{n}\left(f_{V}, f_{W}\right) \stackrel{d}{\longrightarrow} C^{n+1}\left(f_{V}, f_{W}\right) \rightarrow \ldots
$$

The excision axiom holds automatically while the obvious exact sequence

$$
0 \rightarrow C^{n}\left(f_{V}, f_{W}\right) \rightarrow C^{n}\left(f_{V}\right) \rightarrow C^{n}\left(f_{\tilde{W}}\right) \rightarrow 0
$$

implies the long exact sequence

$$
\cdots \rightarrow \hat{H}_{\mathcal{Q}}^{n}\left(f_{V}\right) \rightarrow \hat{H}_{\mathcal{Q}}^{n}\left(f_{W}\right) \rightarrow \hat{H}_{\mathcal{Q}}^{n+1}\left(f_{V}, f_{W}\right) \rightarrow \hat{H}_{\mathcal{Q}}^{n+1}\left(f_{V}\right) \rightarrow \cdots
$$

If $V=\{v\}$ is a point, then $\hat{H}_{\mathcal{Q}}\left(f_{\{v\}}\right)=H_{\mathcal{Q}}\left(f_{\{v\}}\right)$ since the spectral sequence $E_{i, j}^{r}$ degenerates in the page $E_{i, j}^{2}$ in this case.

The homotopy property is automatic for homotopies in the class of functions in the general position. This property is not at all trivial for homotopies that include functions not in general position. Moreover, this property is actually the central point of the whole story; we prove it in the next section.

Remark To be precise, we have to remind that our cochain spaces depend on a small parameter $\varepsilon$. Of course, we simply take $\varepsilon$ smaller each time it is necessary to guarantee that the final result does not depend on $\varepsilon$.

\section{Surgery}

Let $V$ be a manifold with a convex boundary and $f: V \times \mathbb{R} P^{N} \rightarrow \mathbb{R}$ a $C^{1}$-function such that $f_{v} \in \mathcal{Q}, \forall v \in V$. The function $f$ is regular if and only if for any $(v, \bar{x}) \in$ $V \times \mathbb{R} P^{N}$ such that $x \in \operatorname{ker} f_{v}$ there exists $\xi \in T_{v} V$ such that $\left\langle\frac{\partial f}{\partial v}(v, \bar{x}), \xi\right\rangle>0$.

We say that $f$ is strongly regular if for any $v \in V$ such that ker $f_{v} \neq 0$ there exists $\xi \in T_{v} V$ such that $\left\langle\frac{\partial f}{\partial v}(v, \bar{x}), \xi\right\rangle>0$ for any $x \in \operatorname{ker} f_{v} \cap S^{N}$. 
In other words, for the regularity to be strong we ask for $\xi$ in the inequality to be one and the same for all $x \in \operatorname{ker} f_{v} \cap S^{N}$. Here is a typical example of a regular but not strongly regular map:

$$
V=\{q \in \mathcal{Q}: \operatorname{tr} q=0,|q| \leq 1\}, \quad f(q, \bar{x})=q(x) .
$$

Here and below we use the following notations: $\operatorname{tr} q$ is the trace of the symmetric operator on $\mathbb{R}^{N+1}$ associated to $q,\left\langle q_{1}, q_{2}\right\rangle$ is the trace of the product of the operators associated to $q_{1}$ and $q_{2},|q|=\sqrt{\langle q, q\rangle}$. Strong regularity is violated at $q=0$.

Lemma 3 If $f \in \mathfrak{F}_{\mathcal{Q}}$ is in general position, then $f$ is strongly regular.

Proof Let $q \in \mathcal{Q}$ and $\operatorname{ker} q \neq 0$; then $q \in \Lambda_{j, m}^{0}$ for some $j, m$. It is easy to see that $T_{q} \Lambda_{j, m}^{0}$ is the kernel of the linear map $\left.q^{\prime} \mapsto q^{\prime}\right|_{\text {ker } q}, q^{\prime} \in \mathcal{Q}$. Hence the transversality of the map $v^{\prime} \mapsto f_{v^{\prime}}, v^{\prime} \in V$, to $\Lambda_{j, m}^{0}$ at $v \in V$ is equivalent to the surjectivity of the map $\left.\xi \mapsto\left\langle\frac{\partial f}{\partial v}(v, \cdot), \xi\right\rangle\right|_{\operatorname{ker} f_{v}}, \xi \in T_{v} V$, and implies the existence of $\xi \in T_{v} V$ such that the quadratic form $\left\langle\frac{\partial f}{\partial v}(v, \cdot), \xi\right\rangle$ is positive definite on ker $f_{v}$.

Remark We actually proved more than stated: for $f$ to be strongly regular it is sufficient that the map $v \mapsto f_{v}, v \in M$ is transversal to submanifolds $\Lambda_{j, m}^{0}$; transversality to $\Lambda_{j, m}$ is not necessary.

We say that a regular homotopy $f_{t}, t \in[0,1]$, is strongly regular if all $f_{t}$ are strongly regular. Example: take $f$ as in $(5), \alpha \in[0,1)$ and the homotopy $f_{t}=f+t-\alpha$; then $f_{t}$ is strongly regular for all $t$ except of $t=\alpha$. We'll show later that this example is in a sense a universal model of a generic regular but not strongly regular homotopy.

Lemma 4 Assume that $f_{t} \in \mathfrak{F}_{\mathcal{Q}}, f_{t}: V \times \mathbb{R} P^{N} \rightarrow \mathbb{R}, t \in[0,1]$, is a strongly regular homotopy. Then there exists a smooth family of diffeomorphisms ${ }^{2} F_{t}: V \rightarrow V$, such that $F_{0}=i d, F_{t}\left(V_{f_{0}}^{j}\right) \subset V_{f_{t}}^{j}, \forall t \in[0,1], j=1, \ldots, N+1$.

Proof The proof is similar to the proof of Lemma 1. It is sufficient to find a smooth vector field $X_{t}$ on $V$ such that the equality $\lambda_{j}\left(f_{t v}\right)=0$ implies:

$$
\left\langle\frac{\partial f_{t}}{\partial v}(v, \bar{x}), X_{t}(v)\right\rangle>0, \quad \forall x \in \operatorname{ker} f_{t v} \cap S^{N} .
$$

Indeed, fix $t$ and $v$ and consider a trajectory $v(\tau)$ of the flow generated by the field $X_{\tau}$ such that $v(t)=v$. Inequality (6) implies that for any smaller than $t$ and sufficiently close to $t$ number $\tau$ the quadratic form $f_{\tau v(\tau)}$ is negative definite on the linear hull of the eigenvectors of the form $f_{t v}$ corresponding to the eigenvalues $\lambda_{j}\left(f_{t v}\right), \ldots \lambda_{N+1}\left(f_{t v}\right)$. Hence $\lambda_{j}\left(f_{\tau v(\tau)}\right)<0$, according to the minimax principle for the eigenvalues of a symmetric operator. We obtain that any trajectory started in $V_{f_{0}}^{j}$ stays in $V_{f_{t}}^{j}$ for all $t \in[0,1]$.

The existence of a desired vector field is guaranteed by the strong regularity assumption.

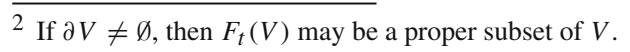


Lemma 3 immediately implies the following:

Corollary 2 Strongly regular homotopies preserve the page $E_{i, j}^{2}$ of the spectral sequence $E_{i, j}^{r}$ described in Sect. 5.

A routine transversality technique gives the following:

Proposition 6 Let $\tilde{f}_{t} \in \mathfrak{F}_{\mathcal{Q}}, \tilde{f}_{t}: V \times \mathcal{Q} \rightarrow \mathbb{R}, t \in[0,1]$ be a regular homotopy and $\tilde{f}_{0}, \tilde{f}_{1}$ are in the general position. Then there exists an arbitrarly $C^{0}$-close to $\tilde{f}_{t}$ regular homotopy $f_{t}$ such that $f_{0}=\tilde{f}_{0}, f_{1}=\tilde{f}_{1}$; the function $f_{t} \in \mathfrak{F}_{\mathcal{Q}}$ is not in general position only for a finite number of values of the parameter $t \in(0,1)$, and for any $f_{t}$ that is not in the general position there exists exactly one point $v_{t}$ where the map $v \mapsto f_{t v}, v \in V$, is not transversal to a submanifold $\Lambda_{j, m}$ or $\Lambda_{j, m}^{0}$ Moreover, if $v_{t} \in$ int $V, f_{t v_{t}} \in \Lambda_{j, m}^{0}$ and the map $v \mapsto f_{t v}, v \in V$, is not transversal to $\Lambda_{j, m}^{0}$ at $v_{t}$, then the following conditions are satisfied:

- The image of the linear map $\left.\frac{\partial f_{t}}{\partial v}\left(v_{t}, \cdot\right)\right|_{\operatorname{ker} f_{t v_{t}}}$ from $T_{v_{t}} V$ into the space of quadratic forms on $\operatorname{ker} f_{t_{v}}$ is a subspace of codimension 1 in the space of quadratic forms and the orthogonal complement to this subspace is generated by $\left.\frac{\partial}{\partial \tau}\right|_{\tau=t}\left(\left.f_{\tau v_{t}}\right|_{\operatorname{ker} f_{t_{t}}}\right)$.

- $\left.\frac{\partial}{\partial \tau}\right|_{\tau=t}\left(\left.f_{\tau v_{t}}\right|_{\operatorname{ker} f_{t_{t}}}\right)$ is a nondegenerate quadratic form.

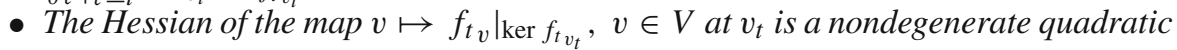
form on the kernel of the map $\left.\frac{\partial f_{t}}{\partial v}\left(v_{t}, \cdot\right)\right|_{\operatorname{ker} f_{t_{v}}}$.

If $v_{t} \in \partial V$ and the map $v \mapsto f_{v}, v \in \partial V$, is not transversal to $\Lambda_{j, m}^{0}$, then the same conditions are satisfied for $f_{\tau \partial V}$ in place of $f_{\tau}$, and the linear map $\left.\frac{\partial f_{t}}{\partial v}\left(v_{t}, \cdot\right)\right|_{\operatorname{ker} f_{t_{t}}}$ from span $T_{v_{t}} V$ into the space of quadratic forms on $\operatorname{ker} f_{t_{t}}$ is surjective.

We are now ready to state a local version of the homotopy invariance property.

Proposition 7 In the setting of Proposition 6, let $t \in(0,1)$ be such that the map $v \mapsto f_{t v}, v \in V$, is not in general position. Then there exist a neighborhood $O_{v_{t}}$ of $v_{t}$ in $V$ and a neighborhood $o_{t}$ of $t$ in $(0,1)$ such that the inclusions $\{\tau\} \times O_{v_{t}} \hookrightarrow o_{t} \times$ $O_{v_{t}}, \tau \in o_{t}$, induce isomorphisms $\hat{H}_{\mathcal{Q}}\left(F_{o_{t} \times O_{v_{t}}}\right) \cong \hat{H}_{\mathcal{Q}}\left(f_{\tau O_{t}}\right)$, where $F_{(\tau, v)} \doteq f_{\tau v}$.

The general "global" homotopy invariance property easily follows from Proposition 7. Indeed, a singularity at $\left(t, v_{t}\right)$ does not influence relative cohomologies for the pairs $\left([0,1] \times V, o_{t} \times O_{v_{t}}\right),\left(V, O_{v_{t}}\right)$ and the inclusion

$$
\left(\{\tau\} \times V,\{\tau\} \times O_{v_{t}}\right) \hookrightarrow\left(o_{t} \times V, o_{t} \times O_{v_{t}}\right)
$$

induces an isomorphism $\hat{H}_{\mathcal{Q}}\left(F_{O_{t} \times V}, F_{o_{t} \times O_{v_{t}}}\right) \cong \hat{H}_{\mathcal{Q}}\left(f_{\tau V}, f_{\tau O_{t}}\right)$. The exact sequences of the pairs $\left(F_{o_{t} \times V}, F_{o_{t} \times O_{v_{t}}}\right),\left(f_{\tau V}, f_{\tau} O_{t}\right)$ and the five lemma imply that the inclusion $\{\tau\} \times V \hookrightarrow o_{t} \times V$ induces an isomorphism $\hat{H}_{\mathcal{Q}}\left(F_{o_{t} \times V}\right) \cong \hat{H}_{\mathcal{Q}}\left(f_{\tau}\right)$.

Proof First assume that the map $v \mapsto f_{t v}, v \in V$, is transversal to all submanifolds $\Lambda_{j, m}^{0}$. Then $f_{t}$ is strongly regular (see the Remark after Lemma 3). Hence $\tau \mapsto$ $f_{\tau} O_{v_{t}}, \tau \in o_{t}$, is a strongly regular homotopy for appropriate neighborhoods $O_{v_{t}}, o_{t}$. 
Moreover, for any $\tau_{0} \in o_{t}$ the maps $(\tau, v) \mapsto f_{\tau v}$ and $(\tau, v) \mapsto f_{\tau_{0} v}$ on $o_{t} \times O_{v_{t}}$ are strongly regular homotopic. Hence $F_{o_{t} \times O_{v_{t}}}$ and $f_{\tau_{0} O_{v_{t}}}$ have equal pages $E_{i, j}^{2}$.

On the other hand, $F_{O_{t}} \times O_{v_{t}}$ is regularly homotopic to a constant family $(\tau, v) \mapsto$ $f_{t v_{t}}+\varepsilon$ according to the general localization result of Sect. 3. Moreover, this regular homotopy is strongly regular in the case under consideration and preserves the page $E_{i, j}^{2}$. The page $E_{i, j}^{2}$ of the constant family has only one nonzero column and the same is true for the families $F_{o_{t} \times O_{v_{t}}}$ and $f_{\tau_{0} O_{v_{t}}}$. In particular, $E_{i, j}^{2}=E_{i, j}^{\infty}$ are equal fot these families.

It remains to study the case when $f_{t v_{t}} \in \Lambda_{j, m}^{0}$ and the map $v \mapsto f_{t v}, v \in V$, is not transversal to $\Lambda_{j, m}^{0}$ at $v_{t}$. Of course it is sufficient to prove the isomorphism $\hat{H}_{\mathcal{Q}}\left(F_{O_{t} \times O_{v_{t}}}\right) \cong \hat{H}_{\mathcal{Q}}\left(f_{\tau O_{v_{\tau}}}\right)$ for one particular $\tau$ greater than $t$ and one $\tau$ smaller than $t$.

We denote by $Q_{t}$ the space of quadratic forms on $\operatorname{ker} f_{v_{t}}, Q_{t}=\mathcal{Q}(m-1)$. Given $q \in \mathcal{Q}$, let $E_{q} \subset \mathbb{R}^{N+1}$ be the linear hull of the eigenvectors of $q$ corresponding to the eigenvalues $\lambda_{j}(q), \ldots, \lambda_{j+m-1}(q)$ and $\pi_{q}: E_{q} \rightarrow \operatorname{ker} f_{v_{t}}$ be the restriction to $E_{q}$ of the orthogonal projector of $\mathbb{R}^{N+1}$ on ker $f_{v_{t}}$. Note that $E_{f_{v_{t}}}=\operatorname{ker} f_{v_{t}}$ and $\pi_{f_{v_{t}}}=i d$. We work in a small neighborhood of $f_{v_{t}}$ in $\mathcal{Q}$ and may assume that $E_{q}$ is transversal to the orthogonal complement of ker $f_{v_{t}}$ and $\pi_{q}$ is invertible.

Consider a map $\Phi: q \mapsto q \circ \pi_{q}^{-1}$ from a neighborhood of $f_{v_{t}}$ to $Q_{t}$. It is a rational map and its differential at the point $f_{v_{t}}$ sends a form $q$ to $\left.q\right|_{\text {ker } f_{v_{t}}}$. Hence $\Phi$ is a submersion of a neighborhood of $f_{v_{t}}$ on a neighborhood of the origin in $Q_{t}$. Moreover, $\lambda_{i}(\Phi(q))=\lambda_{j+i-1}(q), i=1, \ldots, m$.

We take a sufficiently small neighborhood $O_{v_{t}}$ of $v_{t}$ in $V$, a parameter $\tau \in[0,1]$ close to $t$, and define $g_{\tau}: O_{v_{t}} \rightarrow \mathbb{R}$ by the formula: $g_{\tau_{v}}=\Phi\left(f_{\tau_{v}}\right)$. Then $g_{\tau} \in \mathfrak{F}_{Q_{t}}$ and the following equalities are valid ${ }^{3}$ :

$$
V_{g_{\tau}}^{i}=V_{f_{\tau}}^{i+j-1} \cap O_{v_{t}}, \quad g_{\tau}^{-1}\left(\Lambda_{i, k}\right)=f_{\tau}^{-1}\left(\Lambda_{i+j-1, k}\right) \cap O_{v_{t}}
$$

$i=1, \ldots, m-1, k=2, \ldots, n-i+1$. Moreover, $O_{v_{t}} \subset V_{f}^{j-1}, O_{v_{t}} \cap V_{f}^{j+m}=\emptyset$.

It follows that the statement of Proposition 7 for $f_{\tau} \in \mathfrak{F}_{\mathcal{Q}}$ is equivalent to the same statement for $g_{\tau} \in \mathfrak{F}_{Q_{\tau}}$.

We have: $g_{\tau_{v_{t}}}=0$. The family $G:(\tau, v) \mapsto g_{\tau v},(\tau, v) \in o_{t} \times O_{v_{t}}$ is in general position and is strongly regular homotopic to a constant family $(\tau, v) \mapsto c, c>0$, if $o_{t}$ and $O_{v_{t}}$ are sufficiently small. Hence $\hat{H}_{q_{t}}\left(G_{o_{t} \times O_{v_{t}}}\right)=0$.

In what follows, we tacitly substitute $o_{t}$ and $O_{v_{t}}$ by smaller neighborhoods each time it is necessary without changing notations. First we study the case $v_{t} \in$ int $V$ and then explain how the case $v_{t} \in \partial V$ is reduced to the previous one.

To go ahead we need convenient coordinates in $O_{v_{t}}$. We put coordinates on $O_{v_{t}}$ as the product of two balls, $O_{v_{t}}=U \times B=\{(u, q): u \in U, q \in B\}$, where $U \subset \operatorname{ker} \frac{\partial g_{t}\left(v_{t}\right)}{\partial v}, B \subset \operatorname{im} \frac{\partial g_{t}\left(v_{t}\right)}{\partial v}$, in such a way that $v_{t}=(0,0)$ in our coordinates and

$\overline{3 \text { For simplicity, we keep symbol }} g_{\tau}$ for the map $v \mapsto g_{\tau}$ as in Sect. 5 . 


$$
\frac{\partial g_{t}\left(v_{t}\right)}{\partial v}:(u, q) \mapsto q, \quad u \in \operatorname{ker} \frac{\partial g_{t}\left(v_{t}\right)}{\partial v}, q \in \operatorname{im} \frac{\partial g_{t}\left(v_{t}\right)}{\partial v}
$$

We also set $q_{0}=\left.\frac{\partial g_{\tau}\left(v_{t}\right)}{\partial \tau}\right|_{\tau=t}$. Then $B$ is a ball in the hyperplane $q_{0}^{\perp} \subset Q_{t}$. Recall that $q_{0}$ is a nondegenerate quadratic form. Moreover, we assume that the Hessian of the map $v \mapsto g_{t v}$ at $v_{t}$ is normalized. This means that $\operatorname{ker} \frac{\partial g_{t}\left(v_{t}\right)}{\partial v}=\operatorname{span} U$ is splitted in two subspaces, span $U=\mathbb{R}^{i_{+}} \oplus \mathbb{R}^{i_{-}}$, and

$$
\frac{\partial^{2} g_{t}(0,0)}{\partial u^{2}}(u)=2\left(\left|u_{+}\right|^{2}-\left|u_{-}\right|^{2}\right) q_{0}, \quad u=\left(u_{+}, u_{-}\right) \in U, u_{ \pm} \in \mathbb{R}^{i_{ \pm}}
$$

Now we apply a blow-up procedure with a small parameter $\varepsilon>0$. We set:

$$
\varphi_{s}^{\varepsilon}(u, q)=\frac{1}{\varepsilon^{2}} g_{t+\varepsilon^{2} s}\left(\varepsilon u, \varepsilon^{2} q\right), \quad|s| \leq 1,(u, q) \in U \times B .
$$

Note that the multiplication of a quadratic form by a positive number does not change the signs and multiplicities of the eigenvalues. Hence the spectral sequence $E_{i, j}^{r}$ for $\varphi_{s}^{\varepsilon}$ is equal to one for $\left(g_{\tau}\right)_{(\varepsilon U) \times\left(\varepsilon^{2} B\right)}$ with $\tau=t+\varepsilon^{2} s$. We have:

$$
\varphi_{s}^{\varepsilon}(u, q)=q+\left(\left|u_{+}\right|^{2}-\left|u_{-}\right|^{2}+s\right) q_{0}+O(\varepsilon) .
$$

Now fix parameter $s \neq 0$. If $\varepsilon$ is small enough (how small, depends on $s$ ), then the function $\varphi_{s}^{\varepsilon}$ is homotopic to $\varphi_{s}^{0}$ in the class of functions in the general position.

What remains is to prove that $\hat{H}_{Q_{t}}\left(\varphi_{s}^{0}\right)=0$. The following terminology will be useful: given $\varphi: V \rightarrow Q_{t}, \varphi \in \mathfrak{F}_{Q_{t}}$, and a homotopy retraction $h_{\tau}: V \rightarrow V, \tau \in$ $[0,1]$, we say that $h_{\tau}$ is monotone for $\varphi$ if $V_{\varphi \circ h_{\tau}}^{J} \subset V_{\varphi}^{j}, j=1, \ldots m, \tau \in[0,1]$. The homotopy $\tau \mapsto \varphi \circ h_{\tau}$ induced by a monotone deformation retraction preserves the page $E_{i, j}^{2}, d_{2}$ of the spectral sequence.

We study separately three cases.

1. The quadratic form $q_{0}$ is sign-indefinite. In this case $q_{0}^{\perp}$ contains a positive definite form $\hat{q}$. Moreover, if $s$ is sufficiently small then $\hat{q}+s q_{0}$ is a positive definite form. In this case a deformation retraction $h_{\tau}(u, q)=\left((1-\tau)^{\frac{1}{2}} u, \tau \hat{q}+(1-\tau) q\right)$ is monotone for $\varphi_{s}^{0}$. Indeed,

$$
\varphi_{s}^{0}\left(h_{\tau}(u, q)\right)=\tau\left(\hat{q}+s q_{0}\right)+(1-\tau)\left(q+\left(\left|u_{+}\right|^{2}-\left|u_{-}\right|^{2}+s\right) q_{0}\right) .
$$

The signature of a quadratic form (i.e. the numbers of positive and negative eigenvalues) does not change under a linear change of coordinates in $\mathbb{R}^{m}$, although the eigenvalues do change. Take coordinates such that the form $\hat{q}+s q_{0}$ is represented by a scalar matrix. In these coordinates, eigenvalues of the form (7) are linear functions of $\tau$. We have: $\varphi_{s}^{0}\left(h_{1}(u, q)\right) \equiv \hat{q}+s q_{0}$. Hence $E_{i, j}^{2}=0$. 
2. The quadratic form $s q_{0}$ is positive definite. Then the deformation retraction $h_{\tau}(u, q)=\left((1-\tau)^{\frac{1}{2}} u,(1-\tau) q\right)$ is monotone for $\varphi_{s}^{0}$ and $\varphi_{s}^{0}\left(h_{1}(u, q)\right) \equiv s q_{0}$. Hence $E_{i, j}^{2}=0$.

3. The quadratic form $s q_{0}$ is negative definite. In this case, the page $E_{i, j}^{2}$ is very far from being zero. We already mentioned that the transformation of $Q_{t}$ induced by a linear change of coordinates in $\mathbb{R}^{m}$ does not change the signs of eigenvalues and thus the groups $E_{i, j}^{2}$ of the spectral sequences associated to elements of $\mathfrak{F}_{Q_{t}}$. It is important that the differentials $d_{2}$ do not change as well. The last statement needs a justification since the submanifolds $\Lambda_{j, 2} \subset Q_{t}$ do depend on the choice of coordinates in $\mathbb{R}^{m}$. The differential $d_{2}$ of the spectral sequence $E_{i, j}^{r}$ does not depend on the choice of coordinates because it is equal to the differential $d_{2}$ of the spectral sequence $F_{i, j}^{r}$ constructed in Agrachev and Lerario (2012) (see Sect. 5), and $F_{i, j}^{r}$ is the Leray spectral sequence of a map that respects changes of coordinates.

Now take coordinates in $\mathbb{R}^{m}$ such that the form $q_{0}$ is represented by a scalar matrix. Then $B$ is a ball in the space of symmetric matrices with zero trace. If $q_{0}>0$, then the deformation retraction $\left(u_{+}, u_{-}, q\right) \mapsto\left(u_{+},(1-\tau) u_{-}, q\right), \tau \in[0,1]$, is monotone for $\varphi_{s}^{0}$. Similarly, if $q_{0}<0$, then the deformation retraction $\left(u_{+}, u_{-}, q\right) \mapsto$ $\left((1-\tau) u_{+}, u_{-}, q\right), \tau \in[0,1]$, is monotone.

The next lemma completes the proof of Proposition 7 in the case $v_{t} \in$ int $V$,

Lemma 5 Let $0<s<1$,

$$
U=\left\{u \in \mathbb{R}^{k}:|u|^{2} \leq 2\right\}, \quad \mathbb{B}=\{q \in \mathcal{Q}: \operatorname{tr} q=0,\|q\| \leq 1\},
$$

and the map $\varphi: U \times B \rightarrow \mathcal{Q}, \varphi \in \mathfrak{F}_{\mathcal{Q}}$, is defined by the formula: $\varphi(u, q)=$ $q+|u|^{2}-s$. Then the page $E_{i, j}^{3}$ of the spectral sequence $E_{i, j}^{r}$ associated to $\varphi$ is zero.

Proof We have to prove that the cochain complex $\left(E^{2}, d_{2}\right)$ is exact. It is not at all obvious but it is actually proved in Agrachev (2011), Th. 2. Indeed, let us show that the complex $\left(E^{2}, d_{2}\right)$ can be naturally identified with complex (1) from Agrachev (2011), where $n=N+1$.

We set: $M^{j}=\left\{q \in \mathbb{B}:\|q\|=1, \lambda_{N-j+1}(q) \neq \lambda_{N+1}(q)\right\}$, like in Agrachev (2011) (note that the eigenvalues have the reversed ordering in Agrachev (2011)). Recall that $E_{i, j}^{2}=H^{i}\left(V, V_{\varphi}^{j+1}\right)$, where $V=U \times \mathbb{B}$. A simple homotopy that moves only eigenvalues of symmetric matrices keeping fixed the eigenvectors gives a homotopy equivalence of pairs:

$$
\left(U \times \mathbb{B}, V_{\varphi}^{j+1}\right) \cong\left(U \times \mathbb{B},\left(U \times M^{N-j}\right) \cup(\partial U \times \mathbb{B})\right) .
$$

Hence $E_{i, j}^{2}=H^{i-k}\left(\mathbb{B}, M^{N-j}\right)$; moreover, natural isomorphism of $E_{r, \text {, and }}^{2}$ $H^{-k}\left(\mathbb{B}, M^{N-\cdot}\right)$ transforms $d_{2}$ in the differential of the exact complex (1) from Agrachev (2011).

Let $v_{t} \in \partial V$; we consider the maps $\left.g_{\tau}\right|_{\partial V}$, take appropriate coordinates, and apply the blow-up procedure as we did for $g_{\tau}$ in the case of an interior point $v_{t}$. We arrive to 
the $\operatorname{map} \varphi_{s}^{0}:(u, q) \mapsto q+\left(\left|u_{+}\right|^{2}-\left|u_{-}\right|^{2}+s\right) q_{0}$ extended to $U \times B^{+}$or $U \times B^{-}$, where $B^{ \pm}$is the intersection of a ball in $Q_{t}$ with the half-space $\left\{q \in Q_{t}: \pm\left\langle q, s q_{0}\right\rangle \geq 0\right\}$. We denote these extensions by $\varphi_{s}^{ \pm}$. What remains is to prove that $\hat{H}_{Q_{t}}\left(\varphi_{s}^{ \pm}\right)=0$.

If $s q_{0}$ is not negative definite and $|s|$ is sufficiently small, then simple monotone deformation retractions transform $\varphi_{s}^{ \pm}$into a positive constant. The same is true for $\varphi_{S}^{+}$ with a negative definite $s q_{0}$. The only remaining possibility is $\varphi_{s}^{-}$with a negative definite $s q_{0}$. In this case, a deformation retraction $h_{\tau}(u, q)=\left(u, q-\tau \frac{\left\langle q, q_{0}\right\rangle}{\left|q_{0}\right|^{2}} q_{0}\right), \tau \in$ $[0,1]$, is monotone and transforms $\varphi_{s}^{-}$in the already studied $\varphi_{s}^{0}$ defined on $U \times B$.

Remark We have shown that local disturbance in the page $E^{2}$ caused by a violation of the strong regularity during a regular homotopy is totally calmed in the page $E^{3}$. However, this fact does not imply regular homotopy invariance of $E^{3}$ because the complexes $E^{2}, d_{2}$ do not satisfy the exact sequence "axiom" and invariance of their local cohomologies does not imply invariance of the global ones.

\section{An Example}

Let $\mathbb{H}$ be the quaternion algebra, $\mathbb{H}=\mathbb{R} \oplus \mathbb{R}^{3}$, where $\mathbb{R}$ is the real line and $\mathbb{R}^{3}$ is the space of purely imaginary quaternions, $\mathbb{R}^{3}=\{x \in \mathbb{H}: \bar{x}=-x\}$. We take $a \in \mathbb{R}^{3} \backslash\{0\}$ and consider a quadratic map $\varphi: \mathbb{H} \rightarrow \mathbb{R}^{3}$ defined by the formula $\varphi(x)=\bar{x} a x$. Then $|\varphi(x)|=|a||x|^{2}$. In particular, $\varphi^{-1}(x)=0$. The restriction of $\varphi$ to $S^{3}$ is just adjoint representation of the group SU(2) $=S^{3}$ and a realization of the Hopf bundle $S^{3} \rightarrow S^{2}$. Now consider a family of quadratic forms $\varphi_{p}^{*} \in \mathcal{Q}(3), p \in B^{3}=\left\{p \in \mathbb{R}^{3}:|p| \leq 1\right\}$, where $\varphi_{p}^{*}(x)=\langle p, \varphi(x)\rangle$; then $\varphi^{*} \in \mathfrak{F}_{\mathcal{Q}(3)}, \hat{H}_{\mathcal{Q}(3)}\left(\varphi^{*}\right)=0$.

We have $\mathbb{H}=\mathbb{C} \oplus j \mathbb{C}=\mathbb{C}^{2}$. Quadratic forms $\varphi_{p}^{*}$ are thus real quadratic forms on $\mathbb{C}^{2}$. It is easy to see that they are Hermitian quadratic forms whose Hermitian matrices have zero traces. In other words, $\operatorname{span}\left\{\varphi_{p}^{*}: p \in B^{3}\right\}=i \operatorname{su}(2)$. Eigenspaces of the symmetric operators associated to $\varphi_{p}^{*}$ are complex lines in $\mathbb{R}^{4}$; hence the eigenvalues are double and we have

$$
\begin{gathered}
\lambda_{1}\left(\varphi_{p}^{*}\right)=\lambda_{2}\left(\varphi_{p}^{*}\right)=-\lambda_{3}\left(\varphi_{p}^{*}\right)=-\lambda_{4}\left(\varphi_{p}^{*}\right), \\
V_{\varphi^{*}}^{1}=V_{\varphi^{*}}^{2}=B^{3} \backslash\{0\}, \quad V_{\varphi^{*}}^{3}=V_{\varphi^{*}}^{4}=\emptyset .
\end{gathered}
$$

Let $\varsigma$ be a small quadratic form, then $\phi^{*}-\varsigma$ is regularly homotopic to $\varphi^{*}$ and $\hat{H}_{\mathcal{Q}(3)}\left(\varphi^{*}-\varsigma\right)=0$. Moreover, $\varphi^{*}-\varsigma$ is in general position for almost every $\varsigma$.

Assume that $\varsigma$ is positive definite; then $V_{\varphi^{*}-\varsigma}^{1}, V_{\varphi^{*}-\varsigma}^{2}$ are complements to (small) contractible neighborhoods of $0, V_{\varphi^{*}-\varsigma}^{3}=V_{\varphi^{*}-\varsigma}^{4}=\emptyset$. Indeed, the number of positive eigenvalues of the operator associated to a quadratic form does not depend on the choice of the Euclidean structure. If we choose a form $\frac{1}{\varepsilon} \varsigma$ as the Euclidean structure, then $\lambda_{i}\left(\varphi_{p}^{*}-\varsigma\right)=\lambda_{i}\left(\varphi_{p}^{*}\right)-\varepsilon$.

The page $E^{2}$ of the spectral sequence $E^{r}$ for $\varphi^{*}-\varsigma$ has the form: 


$$
\begin{array}{|c|c|c|c}
\mathbb{Z}_{2} & 0 & 0 & 0 \\
\mathbb{Z}_{2} & 0 & 0 & 0 \\
0 & 0 & 0 & \mathbb{Z}_{2} \\
0 & 0 & 0 & \mathbb{Z}_{2} \\
\hline
\end{array}
$$

Hence the differentials $d_{3}: E_{0, j+1}^{2} \rightarrow E_{3, j-1}^{2}, j=2,3$, are not zero. We are in the situation described in Sect. 5 (see the paragraph with formula $(*)$ and the next paragraph). It follows that the linking number mod 2 of $\left(\varphi^{*}-\varsigma\right)^{-1}\left(\Lambda_{2,2}\right)$ with $\left(\varphi^{*}-\varsigma\right)^{-1}\left(\Lambda_{1,2}\right)$ and with $\left(\varphi^{*}-\varsigma\right)^{-1}\left(\Lambda_{3,2}\right)$ are nonzero.

The Proposition stated in the Introduction can be easily derived from this fact. We start from the case of generic $S_{0}$. First of all, $C_{i}^{S_{0}+t I}=C_{i}^{S_{0}}$ for any scalar matrix $t I$. Hence we may assume that $S_{0}$ is the matrix of a negative definite quadratic form. It is sufficient to compute linking numbers of $C_{2}^{S_{0}}$ with $C_{1}^{S_{0}}$ and with $C_{3}^{S_{0}}$ in a very big ball $\frac{1}{\varepsilon} B^{3}$. Multiplication by $\varepsilon$ transforms $C_{j}^{S_{0}}$ into $C_{j}^{\varepsilon S_{0}}=\left(\varphi^{*}-\varsigma\right)^{-1}\left(\Lambda_{j, 2}\right), \quad j=1,2,3$, where $\varsigma$ is the quadratic form represented by the matrix $-\varepsilon S_{0}$.

We have proved the statement about linking numbers in the case of generic $S_{0}$. Now take any $S_{0}$ and present it as the limit of a sequence of generic ones, $S_{0}=\lim _{n \rightarrow \infty} S_{0}^{n}$. Any limiting point of the sequence of sets $C_{j}^{S_{0}^{n}}$ as $n \rightarrow \infty$ belongs to $C_{j}^{S_{0}}$. The curves $C_{2}^{S_{0}^{n}}$ are uniformly bounded, hence $C_{2}^{S_{0}} \neq \varnothing$. The curves $C_{1}^{S_{0}^{n}}$ and $C_{3}^{S_{0}^{n}}$ are linked with $C_{2}^{S_{0}^{n}}$ and cannot escape to infinity; hence $C_{1}^{S_{0}}$ and $C_{3}^{S_{0}}$ are also nonempty.

\section{Informal Discussion}

The anonymous referee asked me to say more about global features of the Lagrange multipliers even if we do not have yet a general conventional theory. Indeed, Arnold journal encourages informal discussions, and I'll try to do it.

Let $F: U \rightarrow M$ be a smooth map from one smooth manifold to another one. Given a critical point $u \in U$ of this map, a Lagrange multiplier is a nonzero covector $\lambda \in T_{F(u)}^{*} M$, which annihilates the image of the differential $D_{x} F: T_{u} U \rightarrow T_{F(u)} M$. In other words, Lagrange multipliers are solutions of the equation $\lambda D_{u} F=0$ where the pair $(\lambda, u)$ is taken from the total space of the vector bundle $F^{*}\left(T^{*} M\right)$ with a removed zero section. The equation is homogeneous on the fibers of the bundle.

The traditional nonhomogeneous "affine" version of this equation concerns the case $M=\mathbb{R} \times N, F=(\varphi, \Phi)$, where $\phi: U \rightarrow \mathbb{R}$ is treated as a "functional" and $\Phi: U \rightarrow M$ defines constraints. The Lagrange multiplier is now an element of $T_{F(u)}^{*}(\mathbb{R} \times N)=\mathbb{R} \oplus T_{\Phi(u)}^{*} N$. Let $u$ be a regular point of $\Phi$; then $u$ is critical for $F$ if and only if it is a critical point of $\varphi$ restricted to the level set of $\Phi$. The first (scalar) component of the Lagrange multiplier does not vanish in this case and can be normalized. We set this scalar to be equal to $(-1)$ and obtain the equation: $\lambda D_{u} \Phi=d_{u} \varphi, \lambda \in T_{\Phi(u)}^{*} M$. The pair $(\lambda, u)$ belongs to $\Phi^{*}\left(T^{*} M\right)$ and $\lambda$ is also called the Lagrange multiplier. Both homogeneous and "affine" versions can be treated similarly. 
The map $(\lambda, u) \mapsto \lambda D_{u} F$ is transversal to the zero section of $F^{*}\left(T^{*} M\right)$ for generic $F$. If it is transversal then we say that $F$ is a Morse map. Indeed, for $M=\mathbb{R}$ this just a usual Morse function. For a Morse map $F$, solutions of the equation $\lambda D_{u} F=0$ form a smooth $(\operatorname{dim} M)$-dimensional submanifold $C_{F}$ of $F^{*}\left(T^{*} M\right)$ (or a $(\operatorname{dim} M-1)$ dimensional submanifold of the projectivization of this vector bundle).

In other words, Lagrange multipliers resolve singularities of the set of critical points. Moreover, the map $F^{c}:(\lambda, u) \mapsto \lambda,(\lambda, u) \in C_{F}$ is a Lagrangian immersion of $C_{F}$ into the manifold $T^{*} M$ endowed with the standard symplectic structure. Similarly for the affine version, and all that is almost a tautology (see Agrachev and Gamkrelidze 1998 for some details). I find it wonderful that Lagrange multipliers form a Lagrange submanifold! Both objects are named after Lagrange but they look very different at the first glance.

The idea is to recover interesting homological invariants of $F$ in terms of the Lagrange multipliers sitting in $T^{*} M$. We would like to develop a theory, which is efficient when $M$ has a modest dimension while $U$ can be huge. The applications most interesting for us concern constrained variational problems where $U$ is an infinite dimensional Hilbert or Banach manifold.

The results of this paper can be easily interpreted as a desired theory for homogeneous quadratic maps. Why do we think that a good theory can be developed in the general setting as well? To any $(\lambda, u) \in C_{F}$ we associate the Hessian $\lambda \operatorname{Hess}_{u} F: \operatorname{ker} D_{u} F \rightarrow \mathbb{R}$ that is a quadratic form on $\operatorname{ker} D_{u} F$. If $M=\mathbb{R}$ then critical points of $F$ are isolated, the Hessians of $F$ at these points are nondegenerate quadratic forms and inertia indices of these quadratic forms are crucial local invariants used by the Morse theory to estimate homology of the Lebesgue sets and level sets of $F$. If $\operatorname{dim} M>1$ then critical points are not isolated and $\lambda \mathrm{Hess}_{u} F$ may be degenerate for some $(\lambda, u) \in C_{F}$.

There is an important duality between the quadratic form $\lambda \operatorname{Hess}_{u} F$ and the image of the tangent space $T_{(\lambda, u)} C_{F}$ under the Lagrangian immersion $F^{c}:(\lambda, u) \mapsto \lambda$. Let $J_{\lambda}=F_{*}^{c}\left(T_{(\lambda, u)} C_{F}\right)$ and $\pi: T^{*} M \rightarrow M$ be the canonical projection. It is easy to check that $\lambda \operatorname{Hess}_{u} F$ is degenerate if and only if $\left.\pi_{*}\right|_{J_{\lambda}}$ is degenerate and dim ker $\lambda \operatorname{Hess}_{u} F=$ $\operatorname{dim} \operatorname{ker}\left(\left.\pi_{*}\right|_{\lambda}\right)$. Moreover, for any continuous curve $\left(\lambda_{t}, u_{t}\right) \in C_{F}, t \in[0,1]$, such that $\lambda_{0} \operatorname{Hess}_{u_{0}} F$ and $\lambda_{1} \operatorname{Hess}_{u_{1}} F$ are nondegenerate, the difference of inertia indices of these quadratic forms is equal to the Arnold-Maslov index of the curve $t \mapsto J_{\lambda_{t}}$. In other words, Arnold-Maslov cocycle of the Lagrangian immersion equals the coboundary of of the inertia index of the Hessian.

It is natural to expect that homological invariants of the Lagrangian immersion properly glue together the Hessians corresponding to different points of one and the same connected component of $C_{F}$ to give such a connected component the role played by the isolated critical point in the usual Morse theory.

The framework is indeed rather similar to one studied in this paper. Let $\mathbf{L}_{\lambda}$ be the Lagrange Grassmannian of all Lagrangian subspaces of the symplectic space $T_{\lambda}\left(T^{*} M\right)$. This Lagrange Grassmannian has a distiguished element $\Pi_{\lambda}=T_{\lambda}\left(T_{\pi(\lambda)}^{*} M\right)$ (the tangent space to the fiber) and is, actually, a natural compactification of the space of quadratic forms on $\Pi_{\lambda}$ (see, for instance, Arnold (1985) or Agrachev and Gamkrelidze 1998). The subspace $J_{\lambda}$ is also an element of $\mathbf{L}_{\lambda}$. 
Given $\Lambda \in \mathbf{L}_{\lambda}$, we have: $\left.\operatorname{ker} \pi_{*}\right|_{\Lambda}=\Lambda \cap \Pi_{\lambda}$. The set of all Lagrangian subspaces which have a nontrivial intersection with $\Pi_{\lambda}$ is called "the train of $\Pi_{\lambda}$ ". So the Hessian changes its inertia index exactly when $J_{\lambda}$ passes the train. On the other hand, the train is the compactification of the space of degenerate quadratic forms on $\Pi_{\lambda}$ (see Arnold 1985). It looks like we always speak about one and the same story....

Acknowledgments I am grateful to Antonio Lerario for stimulating discussions.

\section{References}

Arnold, V.I.: Sturms theorem and symplectic geometry. Funct. Anal. Appl. 19, 114 (1985)

Agrachev, A., Gamkrelidze, R.: Symplectic methods in optimization and control. In: Jakubczyk, B., Respondek, W. (eds.) Geometry of Feedback and Optimal Control. Marcel Dekker, New York $(1998,1977)$

Agrachev, A.: On the space of symmetric operators with multiple ground states. Funct. Anal. Appl. 45, 241-251 (2011)

Agrachev, A., Lerario, A.: Systems of quadratic inequalities. Proc. Lond. Math. Soc. 105, 622-660 (2012)

Eilenberg, S., Steenrod, N. E.: Foundations of Algebraic Topology. Princeton University Press, Princeton, $\mathrm{XV}+328 \mathrm{pp}(1952)$

Kurdyka, K.: On gradients of functions definable in o-minimal structures. Ann. Inst. Fourier 48, 769-783 (1998)

McCleary, J.: A User's Guide to Spectral Sequences, p. 578. Cambridge University Press, Cambridge (2001) Morse, M.: The calculus of variations in the large, vol. 18, pp. 64-70. American Mathematical Society Colloquium Publications, New York (1934) 